\title{
Einsamkeit: der unerkannte Killer
}

\section{Anne-Françoise Allaz}

Prof. Dr. med., Mitglied der Redaktion

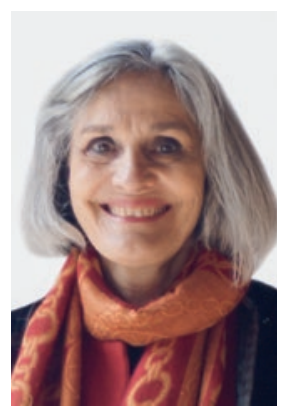

Literatur

1 Berhuet S, Mansencal LB, Etienne L, et al. Dix ans d'observation de l'isolement relation nel: un phénomène en forte progression. Les solitudes en France-Fondation de France, 2020.

2 Schweizerische Gesundheitsbefragung. BFS, 2019.

3 Holt-Lunstad J, et al. Social Relationships and Mortality Risk: A Meta-analytic Review. PLoS Med. 2010;7(7):e1000316.

4 Bzdok D, Dunbar R. Neurobiology of Social distancing. Trends in Cognitive Sciences. 2020;24: 717-33.

afallaz[at]bluewin.ch

\author{
«Das Geheimnis eines schönen Alters ist der \\ würdige Umgang mit der Einsamkeit.» \\ G. García Márquez, Hundert Jahre Einsamkeit
}

Laut einer im November 2020 veröffentlichten Umfrage der SRG ist in der Schweiz zwischen Juni und Oktober 2020 die Angst vor sozialer Isolation und Einsamkeit im Zusammenhang mit der Pandemie von 30 auf $46 \%$ gestiegen.

Diese Umfrage spiegelt den jüngsten Bericht der Fondation de France wider, der den Aspekt der «Einsamkeit» [1] über die letzten zehn Jahre hinweg betrachtet. Daraus geht hervor, dass die soziale Isolation zunimmt und dass in Frankreich 15\% der Bevölkerung davon betroffen sind. Von den isolierten Personen bezeichnen sich $38 \%$ als «misstrauisch» oder «leidend», 43\% hingegen als «ausgeglichen» oder «aus freier Entscheidung allein lebend». Soziale Isolation betrifft vor allem Menschen in prekären Situationen oder ältere Menschen, aber auch - und das ist besorgniserregend - zunehmend junge Menschen. In der Schweiz gaben im Jahr 2017 laut einer Erhebung des Bundesamts für Statistik (BFS) 38\% der Wohnbevölkerung und sogar 46\% der Migrantinnen und Migranten der ersten Generation an, einsam zu sein [2].

Soziale Isolation und Einsamkeit hängen miteinander zusammen, sind aber nicht das Gleiche. Soziale Isolation lässt sich an der Häufigkeit der Kontakte und der Einbindung in ein Netzwerk messen. Das Gefühl der Einsamkeit hingegen ist laut BFS «der subjektive Ausdruck eines Mangels an sozialen Ressourcen oder eines Bedürfnisses nach zusätzlichen oder anderweitigen sozialen Kontakten".

Die Covid-Zeit ist geprägt von den Auswirkungen des Verlustes sozialer Bindungen. Doch was wissen wir eigentlich hierüber? Es gibt seit Jahren fundierte Hinweise darauf, dass ein Mangel an sozialer Integration das Risiko vorzeitiger Sterblichkeit, insbesondere aus kardiovaskulären Gründen, signifikant erhöht. «Das Ausmass dieses Risikos übersteigt das vieler klassischer Risikofaktoren", so das Fazit einer Meta-Analyse mit mehr als 300000 Patientinnen und Patienten [3]. Bertrand Kiefer formuliert es in der Revue Médicale Suisse prägnanter: «Einsamkeit ist tödlicher als Fettleibigkeit.» Eine aktuelle Studie ergänzt dieses düstere Bild um Daten über Immunschäden und das Risiko einer Alzheimer-Erkrankung im Zusammenhang mit sozialer Deprivation oder Einsamkeit [4]. Hinzu kommen die bekannteren psychologischen Folgen wie Depressionen und Suizid oder die Auswirkungen auf die Lebensqualität, das Selbstbild oder der Verzicht auf medizinische Versorgung.

Angesichts dieser Daten kann man sich nur darüber wundern, wie wenig dieser wichtige Gesundheitsfaktor thematisiert wird. Dass der Mensch ein durch und durch soziales Wesen ist, wussten wir bereits. Menschen lieben Gesellschaft. Wir brauchen Interaktion, Gespräche, Gefühle und körperlichen Kontakt. Diese Aspekte sind lebenswichtig. Mangelt es uns daran, macht uns dies anfällig für Krankheiten und kann uns sogar das Leben kosten. Denken Sie nur an die erschreckende Übersterblichkeit von Kindern, die ohne Aufmerksamkeit und Zuneigung in Waisenhäusern aufwachsen - ganz zu schweigen von ihren schweren Entwicklungsproblemen.

Doch die soziale Isolation nimmt im gesamten Westen stark zu, weshalb sich die WHO besorgt zeigt. Es gibt bereits Regierungen, die entsprechende Ministerien und Kommissionen ins Leben rufen, um die Einsamkeit zu bekämpfen. So wurde beispielsweise in Grossbritannien eine Stelle geschaffen, die umgangssprachlich mit «Minister for Loneliness» bezeichnet wird. Zahlreiche Institutionen und Vereine engagieren sich für die Bewahrung von sozialen Bindungen und der Solidarität. Was bedeutet das für uns Ärztinnen und Ärzte? Wir stehen gemeinsam mit den Apothekerinnen und Apothekern und den häuslichen Pflegekräften an vorderster Front, was den Kontakt mit isolierten Menschen anbelangt. Daher dürfen wir aus medizinischen und humanistischen Gründen dieser unbequemen Thematik nicht ausweichen. Wenn wir uns des Problems bewusst sind, können wir auf verschiedenen Ebenen reagieren. Sei es durch Einflussnahme auf die Gesundheitspolitik oder durch die Unterstützung von Institutionen, die Kontakte herstellen und zur sozialen Wiedereingliederung beitragen. Aber auch, indem wir Patientinnen und Patienten in Kontakt mit Netzwerken, Vereinen oder Ressourcen bringen, auf die sie in Notlagen zurückgreifen können. All diese scheinbar banalen Massnahmen können die Gesundheit fördern oder sogar Lebenswege beeinflussen. In diesem Sinne wünsche ich Ihnen ein Jahr 2021 voller Solidarität! 